CORRESPONDENCE

\title{
Myeloid malignancies in cancer patients treated with poly(ADP- ribose) polymerase (PARP) inhibitors: a case series
}

(c) The Author(s) 2022

Blood Cancer Journal (2022)12:11; https://doi.org/
10.1038/s41408-022-00607-7

\section{Dear Editor}

Poly (ADP-ribose) polymerase (PARP) inhibitors interact with DNA at single-strand breaks, prevent repair, and generate irreparable double-strand breaks that lead to tumor cell death [1]. Patients who harbor defects in homologous recombination repair, such as those with BRCA mutations, have tumors that are especially sensitive to PARP inhibitors.

However, in their meta-analysis of 28 randomized controlled trials, 18 of which were placebo-controlled, Morice et al. cited the incidence of myeloid malignancies with PARP inhibitors at $0.73 \%$ compared to $0.47 \%$ in controls (odds ratio: 2.63 (95\% confidence interval: 1.13-6.14); $p=0.026$ )) [2]. This risk is small but more than doubled, even after controlling for prior platinum-based chemotherapy.

Here, we report a series of patients with PARP inhibitorassociated myeloid malignancy. Medical records were interrogated for patients prescribed a PARP inhibitor (olaparib, rucaparib, veliparib, niraparib, talazoparib) and cross-referenced against those who underwent a bone marrow (BM) biopsy with a myeloid malignancy. Two hematopathologists independently re-reviewed all available peripheral blood and BM slides and laboratory results including molecular findings from Next Generation Sequencing (NGS). In most patients, molecular testing for myeloid-associated mutations had been performed with the OncoHeme NGS panel which interrogated 42 genes recurrently mutated in myeloid neoplasms [3]. In two patients, an 11-gene panel (CEBPA, DNMT3A, FLT3, IDH1, IDH2, KIT, KRAS, NPM1, NRAS, RUNX1, and TP53) had been applied. Cytogenetic findings were reviewed and reported according to the 2020 International System for Human Cytogenomic Nomenclature [4].

\section{CASES}

Between 2014 and 2020, 583 PARP inhibitor-treated patients were identified. Nine had undergone a BM biopsy that showed myelodysplastic syndrome (MDS) in four and acute myeloid leukemia (AML) in five.

\section{CLINICAL FINDINGS}

All nine were women with breast and gynecologic malignancies, reflective of common indications for these agents (Table 1).

In two patients the cumulative duration of PARP inhibitor therapy was greater than 2 years, and all stopped the PARP inhibitor because of a myeloid neoplasm. The median time from initiation of the PARP inhibitor to diagnosis of a myeloid neoplasm was 19 months (range: 4, 56 months), and, incidentally, from initiation of chemotherapy to the myeloid neoplasm 68 months (range: 16, 121 months). Four patients are deceased with a median survival of 0.9 months (range: 0.9 to 3 months). Among those alive, the median survival was 18 months (range: 11, 35 months).

\section{PERIPHERAL BLOOD AND BM FINDINGS}

In all nine patients, bicytopenia or pancytopenia was observed (Table 2). The four MDS cases (patients 1-4) included one with excess blasts-1, two with ring sideroblasts and multilineage dysplasia, and one with single lineage dysplasia. The five AML cases (patients 5-9) included two with morphologic features of pure erythroid leukemia, one with acute monocytic leukemia, and two with AML not otherwise specified.

The BM was hypercellular in seven cases, normocellular in one MDS case, and hypocellular in one MDS case. Dysmegakaryopoiesis was observed in three of four MDS cases (Table 2) and three of the five AML cases. Abnormally small megakaryocytes with hypolobate nuclei were the predominant morphology. Clear-cut dysgranulopoiesis was less common, observed in only two cases.

Outside of the two pure erythroid leukemia cases (patients 6 and 7), unequivocal dyserythropoiesis was seen in one case. In eight cases in which an iron stain had been performed, ring sideroblasts were detected in two MDS and three AML cases (Table 2).

\section{CYTOGENETICS}

Conventional chromosome analysis showed that all except patient 5 had a complex karyotype (Table 2). Except for patients 4 and 9, six of eight patients with a complex karyotype also met the definition of a monosomal karyotype, as defined by Kayser et al [5]. Among all nine patients, the most common abnormalities were -5 or a structural abnormality that resulted in $5 q-$ and -7 or structural abnormalities resulting in $7 q-$. Loss of $17 p$ was observed in three patients (patients $4,6,8$ ), all of which represented biallelic inactivation of TP53 when investigated further by fluorescence in situ hybridization (FISH) (Table 2).

\section{MOLECULAR GENETICS}

Mutation profiling by a 42-gene NGS panel was performed in two of five AML and in all four MDS cases; in two AML cases (patients $8,9)$, a focused 11-gene panel was employed. Pathogenic alterations involving the TP53 gene were detected in three of four MDS patients and in three of four AML patients (Table 2). Despite ring sideroblasts in patients $1,3,6$, and 7 , no pathogenic alterations of the SF3B1 gene were identified. For patient 8 in whom ring sideroblasts were detected, the smaller 11-gene NGS panel did not include SF3B1.

In all patients with TP53 mutations, we observed double mutations (patients 1 and 3 ), a VAF consistent with loss of 


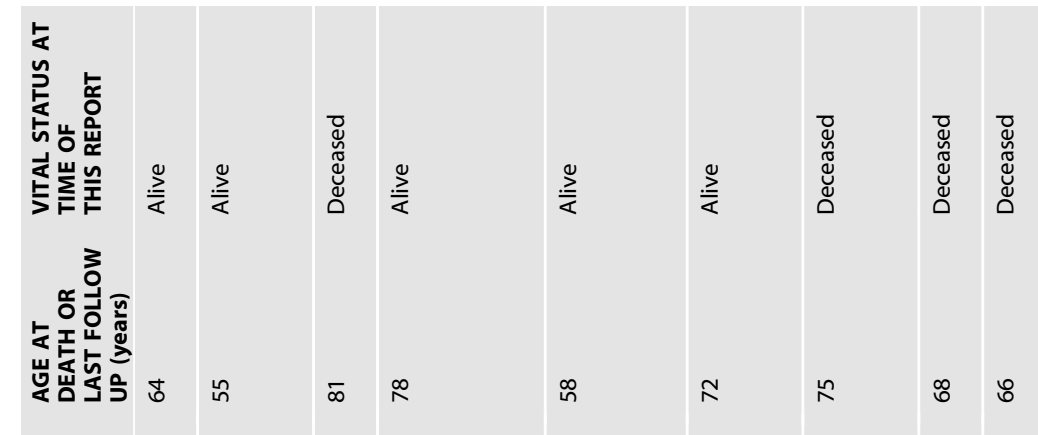

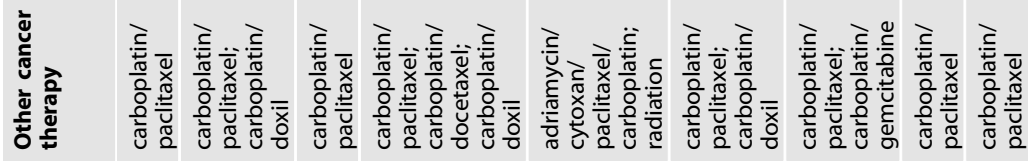

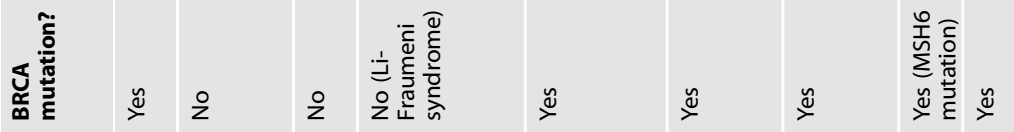

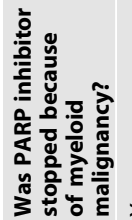

"t.:

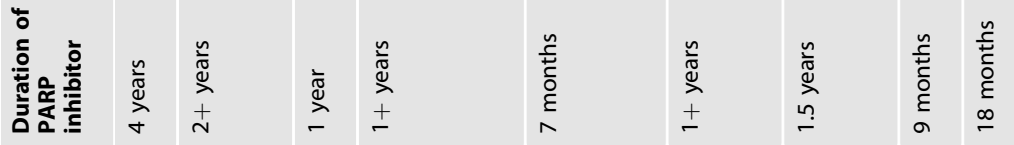

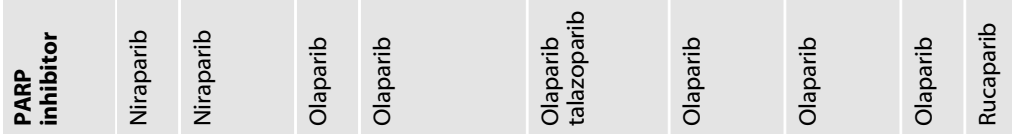

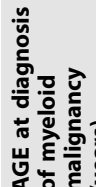

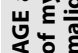

กั

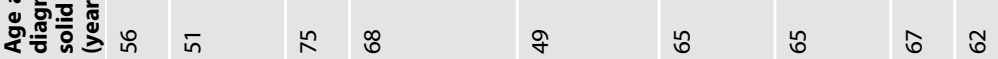

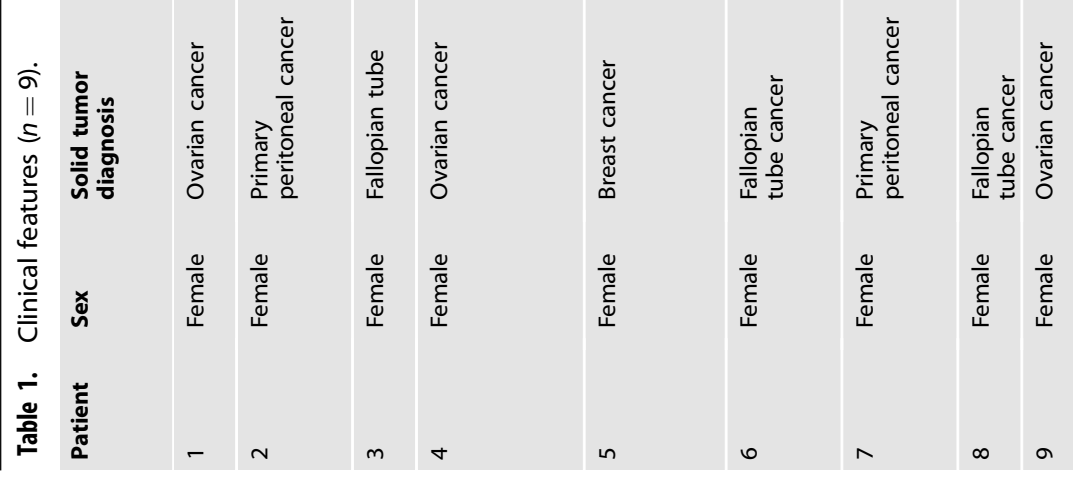




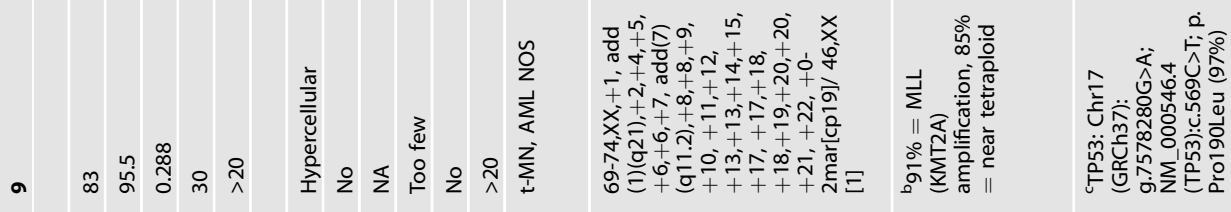

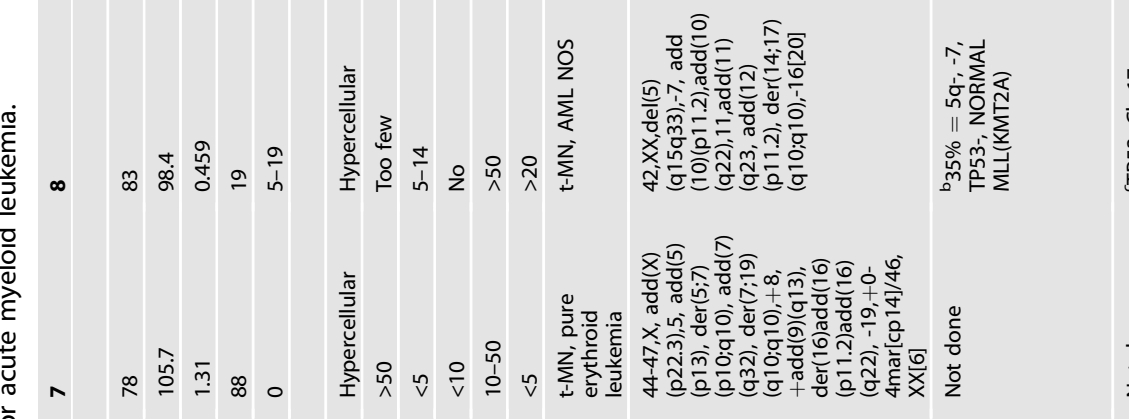

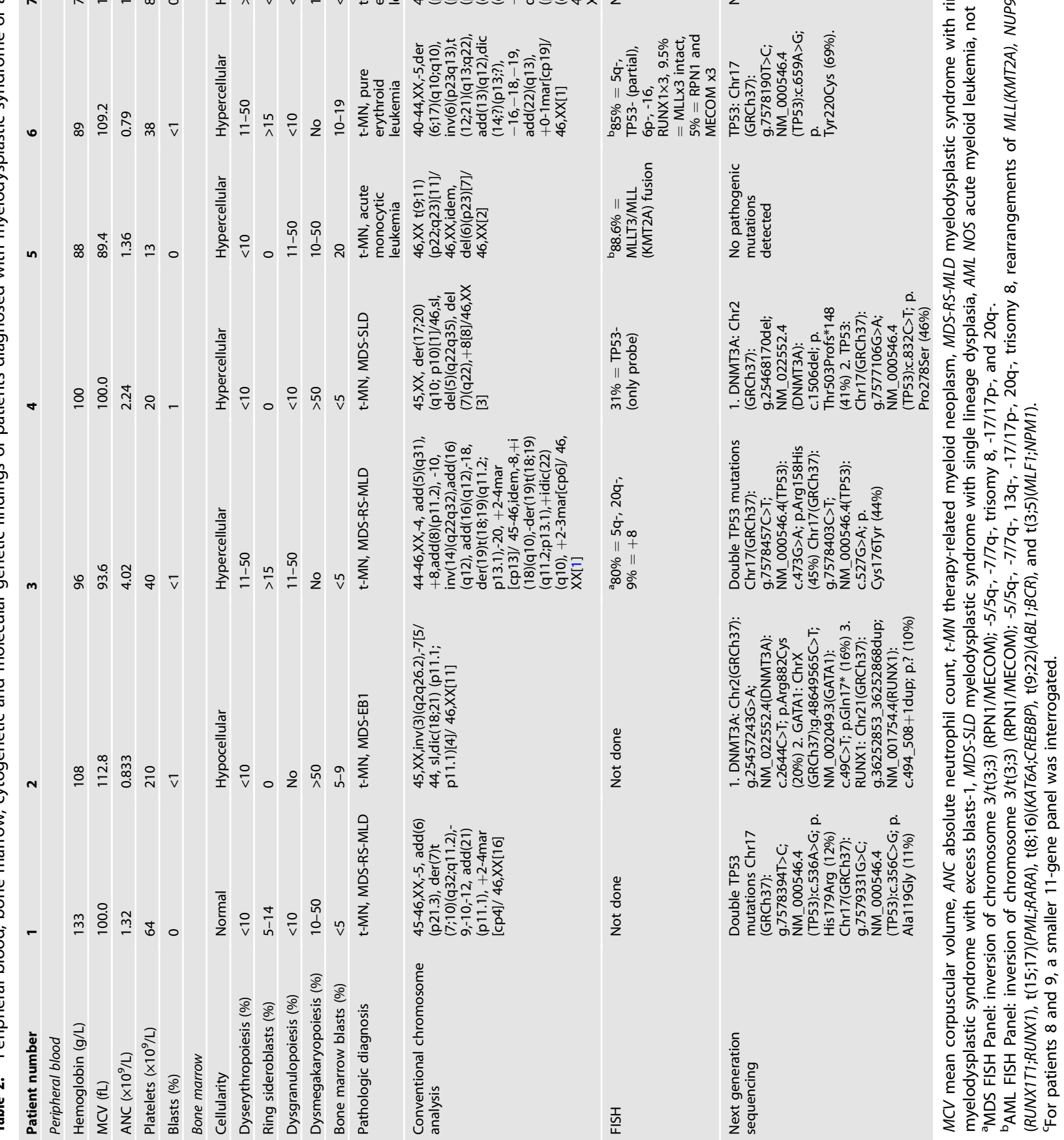


heterozygosity (patient 9), or biallelic inactivation by FISH analysis (patients 4, 6, 8). In five of six patients (patients 1, 3, 6, 8, 9), TP53 was the only gene affected. In patient 4 , a concurrent pathogenic DNMT3A gene alteration was identified. Most TP53 mutations were missense variants in the DNA binding domain with one frameshift variant in the transactivating domain.

To our knowledge, this is the first comprehensive report of the pathologic and genetic characteristics of myeloid malignancies in patients treated with PARP inhibitors. Two findings are especially striking: 1) complex karyotypes with frequent involvement of chromosomes 5 and 7 with a prevalence of a monosomal karyotype and 2) the frequent occurrence of pathogenic TP53 mutations, with TP53 often the sole gene affected either as biallelic inactivation or double mutations. Although dysmegakaryopoiesis was frequently observed, we observed no unique morphologic features in this series. The NGS panel employed for this study did not include PPM1D; otherwise, our observations corroborate the molecular findings reported by Martin and others [6].

Complex karyotypes, frequent TP53 mutations [7, 8] and disconnect between ring sideroblasts and SF3B1 mutations $[9,10]$ distinguish PARP inhibitor-associated myeloid neoplasms from de novo neoplasms [11, 12]. These characteristics seem directly tied to the mechanism of action: PARP inhibitors prevent the repair of DNA single-stranded breaks and thereby give rise to double stranded breaks, so-called synthetic lethality. When these drugs cause major disruptions in genomic stability-often in patients who have BRCA mutations and others prone to such genomic instability-it is not surprising that the myeloid malignancies diagnosed after exposure to a PARP inhibitor demonstrate chromosomal instability and frequent alterations selectively involving the DNA-damage response gene TP53.

Although it is less clear whether PARP inhibitor-associated myeloid neoplasms differ from other therapy-related myeloid neoplasms, three points suggest differences. First, unlike studies of therapy-related myeloid neoplasms in which a median of three gene mutations were observed [11], here, TP53 gene alterations were the only mutated gene in most cases. Second, TP53 gene mutations in $75 \%$ of these patients are higher than the $30-40 \%$ frequency reported in other therapy-related myeloid neoplasms $[11,12]$. Moreover, through biallelic inactivation, loss of heterozygosity, or double TP53 mutations suggestive of biallelic inactivation, the TP53 gene appears at higher risk for inactivation with PARP inhibitors. Although the small number of patients in this series precludes a definitive determination, our findings raise the question of a more central role of TP53 gene alterations in PARP inhibitor-associated myeloid neoplasms, particularly in these types of patients, some of whom harbor BRCA mutations or other underlying defect(s) in DNA repair mechanisms. Third, in contrast to other agents, such as alkylators, which lead to the development of myeloid malignancies within 5-10 years, PARP inhibitors appear to lead to a relatively rapid development of myeloid neoplasms, as suggested by a cumulative exposure to PARP inhibitors of under 5 years and continued use of PARP inhibitor up until the diagnosis of a myeloid neoplasm. These observations suggest that PARP inhibitor-associated myeloid neoplasms merit attention as a specific entity of therapy-related myeloid neoplasms.

This case series has limitations. First, without information on TP53 alterations as clonal hematopoiesis of indeterminate potential (CHIP) mutations prior to PARP inhibitor exposure, we cannot exclude expansion of pre-existing CHIP mutations as a contributor to myeloid neoplasms following PARP inhibitors [13]. Only a longitudinal study could assess whether CHIP or other gene alterations predispose to these myeloid neoplasms. Second, the NGS panels employed were geared towards myeloid cancers with only a limited number of cancer-predisposing genes such as DDX41 and PTPN11 interrogated but not others such as CHEK2 and RTEL1. Given recent observations of a high likelihood of an underlying cancer predisposition germline variant among patients with two or more cancers [14], a more comprehensive germline and somatic genetic analysis beyond BRCA1/ 2 mutation testing might be of value in identifying patients at risk for PARP inhibitor-related myeloid neoplasms. Similarly, we cannot exclude delayed synergistic interactions between PARP inhibitors and prior chemotherapy as the cause of myeloid neoplasms, particularly as others have suggested such therapeutic synergy. Third, 6 patients rapidly transitioned to supportive care alone and 3 had only short follow up after chemotherapy for a myeloid malignancy. Further outcome data would be of value. Fourth, this series generated only nine patients, but the study by Morice and others reported 21 of 4533 patients. Thus, these nine patients -- who underwent in-depth clinical, morphologic, cytogenetic, and molecular genetic assessment - provide instructive findings.

Jennifer L. Oliveira ${ }^{1}$, Patricia T. Greipp ${ }^{2}$, Aruna Rangan ${ }^{1}$, Aminah Jatoi (iD ${ }^{3 \dot{凶}}$ and Phuong L. Nguyen ${ }^{1}$

${ }^{1}$ Division of Hematopathology, Mayo Clinic, Rochester, MN, USA.

${ }^{2}$ Division of Laboratory Genetics and Genomics, Mayo Clinic, Rochester, MN, USA. ${ }^{3}$ Division of Medical Oncology, Mayo Clinic, Rochester, MN, USA. ${ }^{\circledR e m a i l: ~ j a t o i . a m i n a h @ m a y o . e d u ~}$

\section{REFERENCES}

1. Velagapudi UK, Patel BA, Shao X, Pathak SK, Ferraris DV, Talele TT. Recent development in the discovery of PARP inhibitors as anticancer agents: a patent update (2016-2020). Expert Opin Ther Pat. 2021;31:609-23.

2. Morice PM, Leary A, Dolladille C, Chretien B, Poulain L, Gonzalez-Martin A, et al. Myelodysplastic syndrome and acute myeloid leukaemia in patients treated with PARP inhibitors: a safety meta-analysis of randomised controlled trials and a retrospective study of the WHO pharmacovigilance database. Lancet Haematol. 2021;8:e122-e34.

3. He R, Devine DJ, Tu ZJ, Mai M, Chen D, Nguyen PL, et al. Hybridization capturebased next generation sequencing reliably detects FLT3 mutations and classifies FLT3-internal tandem duplication allelic ratio in acute myeloid leukemia: a comparative study to standard fragment analysis. Mod Pathol. 2020;33:334-43.

4. McGowan-Jordan J, Hastings R, Moore S (eds) ISCN 2020: An international system for human cytogenomic nomenclature (Karger Basel, Switzerland 2020).

5. Kayser S, Zucknick M, Dohner K, Krauter J, Kohne CH, Horst HA, et al. Monosomal karyotype in adult acute myeloid leukemia: prognostic impact and outcome after different treatment strategies. Blood. 2012;119:551-8.

6. Martin JE, Khalife-Hachem S, Grinda T, Kfoury M, Garciaz S, Pasquier F, et al. Therapy-related myeloid neoplasms following treatment with PARP inhibitors: new molecular insights. Ann Oncol. 21021;32:1046-8

7. Kuzmanovic T, Patel BJ, Sanikommu SR, Nagata Y, Awada H, Kerr CM, et al. Genomics of therapy-related myeloid neoplasms. Haematologica. 2020;105: e98-e101.

8. Ok CY, Patel KP, Garcia-Manero G, Routbort MJ, Fu B, Tang G, et al. Mutational profiling of therapy-related myelodysplastic syndromes and acute myeloid leukemia by next generation sequencing, a comparison with de novo diseases. Leuk Res. 2015;39:348-54.

9. Malcovati L, Karimi M, Papaemmanuil E, Ambaglio I, Jadersten M, Jansson M, et al. SF3B1 mutation identifies a distinct subset of myelodysplastic syndrome with ring sideroblasts. Blood. 2015;126:233-41.

10. Mangaonkar AA, Lasho TL, Finke CM, Gangat N, Al-Kali A, Elliott MA, et al. Prognostic interaction between bone marrow morphology and SF3B1 and ASXL1 mutations in myelodysplastic syndromes with ring sideroblasts. Blood Cancer J. 2018;8:18.

11. Singhal D, Wee LYA, Kutyna MM, Chhetri R, Geoghegan J, Schreiber AW, et al. The mutational burden of therapy-related myeloid neoplasms is similar to primary myelodysplastic syndrome but has a distinctive distribution. Leukemia. 2019;33:2842-53.

12. Haase D, Stevenson KE, Neuberg D, Maciejewski JP, Nazha A, Sekeres MA, et al. TP53 mutation status divides myelodysplastic syndromes with complex karyotypes into distinct prognostic subgroups. Leukemia. 2019;33:1747-58.

13. Bolton KL, Ptashkin RN, Gao T, Braunstein L, Devlin SM, Kelly D, et al. Cancer therapy shapes the fitness landscape of clonal hematopoiesis. Nat Genet. 2020;52:1219-26.

14. Singhal D, Hahn CN, Feurstein S, Wee LYA, Moma L, Kutyna MM, et al. Targeted gene panels identify a high frequency of pathogenic germline variants in patients diagnosed with a hematoological malignancy and at least one other independent cancer. Leukemia 2021;35:3245-3256. 
ACKNOWLEDGEMENTS

Aminah Jatoi, M.D. is the Betty J. Foust, M.D. and Parents' Professor of Oncology.

\section{AUTHOR CONTRIBUTIONS}

All authors contributed equally to this work.

\section{COMPETING INTERESTS}

The authors declare no competing interests.

\section{ADDITIONAL INFORMATION}

Correspondence and requests for materials should be addressed to Aminah Jatoi.

Reprints and permission information is available at http://www.nature.com/ reprints
Publisher's note Springer Nature remains neutral with regard to jurisdictional claims in published maps and institutional affiliations.
Open Access This article is licensed under a Creative Commons Attribution 4.0 International License, which permits use, sharing, adaptation, distribution and reproduction in any medium or format, as long as you give appropriate credit to the original author(s) and the source, provide a link to the Creative Commons license, and indicate if changes were made. The images or other third party material in this article are included in the article's Creative Commons license, unless indicated otherwise in a credit line to the material. If material is not included in the article's Creative Commons license and your intended use is not permitted by statutory regulation or exceeds the permitted use, you will need to obtain permission directly from the copyright holder. To view a copy of this license, visit http://creativecommons. org/licenses/by/4.0/.

(c) The Author(s) 2022 